\title{
Accuracy of the mean sea level continuous record with future altimetric missions: Jason-3 vs. Sentinel-3a
}

\author{
L. Zawadzki and M. Ablain \\ Collecte Localisation Satellite (CLS), Ramonville-Saint-Agne, France \\ Correspondence to: L. Zawadzki (lzawadzki@cls.fr)
}

Received: 3 June 2015 - Published in Ocean Sci. Discuss.: 17 July 2015

Revised: 17 November 2015 - Accepted: 8 December 2015 - Published: 15 January 2016

\begin{abstract}
The current mean sea level (MSL) continuous record, essential to understanding the climate evolution, is computed with the altimetric measurements of the TOPEX/Poseidon mission, succeeded by Jason-1 and later Jason-2. The accurate continuity of the record is ensured by the conservation of the "historical" TOPEX orbit as well as by calibration phases between the successive missions which enable a rigorous computation of their relative biases. In order to extend the current MSL record, Jason-3 will be the natural successor of Jason-2: on the same orbit with a calibration phase. Shortly after Jason-3, another altimetric climateoriented mission, Sentinel-3a, will be launched on a different orbit. In this paper, simulated altimetric sea level data are used to study the sensitivity of the MSL continuous record to the change of the "historical" orbit for the new Sentinel-3a orbit. By estimating the impact of the absence of calibration phase on the MSL continuous record trend accuracy at the global and regional scales and the impact of the orbit change on the long-term continuity of this MSL record, this study shows that linking Sentinel-3a data instead of Jason-3 to the MSL continuous record would not meet climate user requirements regarding the MSL trend accuracy.
\end{abstract}

\section{Introduction}

Because it integrates changes and interactions of all components of the climate system (ocean, atmosphere, cryosphere, hydrosphere), the estimation of the current sea-level rise is a major indicator of climate change (Ablain et al., 2015; Cazenave, 2004). The accurate monitoring of global mean sea level (GMSL) has been made possible with the development of altimetry missions. Thanks to the uninterrupted succession of TOPEX/Poseidon, Jason-1 and Jason-2 missions on the same orbit, the GMSL has been computed on a continual basis since January 1993. The other altimeter missions (ERS-1, ERS-2, Envisat, SARAL/AltiKa, Geosat Follow-On, Cryosat-2) have been contributing on the one hand to better analyze the long-term stability of the reference missions (e.g., Ollivier et al., 2012, for Envisat) and on the other hand to provide measurements at high latitudes (higher than $66^{\circ} \mathrm{N}$ and S) (Prandi et al., 2012). Besides, combining all these missions with the reference missions allowed a better spatial resolution of sea-level gridded products (AVISO, Dibarboure et al., 2011, SL_cci project, Ablain et al., 2015).

The GMSL time series are therefore only based on TOPEX/Poseidon, Jason-1 and Jason-2 (denoted "reference missions" in the paper) by several prominent groups: AVISO (aviso.altimetry.fr/msl), Colorado University (sealevel.colorado.edu/), GSFC (http://climate.nasa.gov/ vital-signs/sea-level/), NOAA (http://www.star.nesdis.noaa. gov/sod/lsa/SeaLevelRise/), etc. The main advantage of using these continuous data records with three missions on the same orbit comes from the "calibration phases" between TOPEX and Jason-1 (about 7 months in 2002) as well as between Jason-1 and Jason-2 (about 7 months in 2008). During this period, both satellites measured the same sea level spaced about 1 min apart - on the same ground track. This calibration phase is essential to computing accurate sea level relative biases between two missions in order to link their global and regional MSL time series (Leuliette et al., 2004; Dorandeu et al.,2003). This error of the GMSL relative bias estimate between TOPEX and Jason-1 or Jason-1 and Jason2 has been estimated to be close to $0.5-1 \mathrm{~mm}$ (Ablain et al., 2009). For comparison, the error to link TOPEX-A and TOPEX-B (February 1999) - where no overlapping data ex- 
ist between both TOPEX phases - goes up to $2 \mathrm{~mm}$. Although these linking errors seem a priori low, the impact on the GMSL trend has been estimated close to $0.2 \mathrm{~mm} \mathrm{yr}^{-1}$ from 1993 to 2008 in the same study, which is significant with regard to climate user requirements: $0.3 \mathrm{~mm} \mathrm{yr}^{-1}$ over 10 years (GCOS, 2011). This demonstrates the importance of minimizing the linking errors.

In order to extend the current MSL continuous record, the Jason-3 altimetric mission (launch expected in January 2015) will be the natural successor of Jason-2, Jason-1 and TOPEX/Poseidon missions. A similar design as Jason-2, appropriate for climate studies, the same historical ground track as TOPEX, and a calibration phase (at least 7 months) with Jason-2 are the main criteria to justify this a priori choice. In the meantime, another new altimetric mission, Sentinel3a, will be launched (expected in February 2015). One of its main objectives is also to provide accurate sea level measurements for climate studies (Berger et al., 2012; Donlon et al., 2012). Although, the Sentinel-3a ground track will be different from the historical TOPEX one (27-day repeat cycle instead of 9.91), it is relevant to know if it would be possible or not to extend the MSL time series with Sentinel-3a instead of Jason-3, meeting the climate user requirements.

The objective of this paper is to answer this question. In this study, we assumed the error budgets of Jason-2, Jason-3 and Sentinel-3a missions were the same, both for short temporal scales (Philipps et al., 2013) and for the long-term stability (Ablain et al., 2013). The reader's attention is being drawn to the fact that this hypothesis does not take into account the impact of the mean sea surface (MSS) model. Indeed, the error budget must take into account the quality of the MSS model over the mission ground tracks. However, Sentinel-3a is on a new orbit that has never been repetitively sampled. Thus, as long as the MSS model is not reprocessed with Sentinel-3a measurements, the impact in its error budget will be larger than in Jason-2 and Jason-3's (see Dorandeu et al., 2004). In this paper, we did not take this temporary error into account and made the hypothesis of similar error budgets to focus on the impact of the new Sentinel-3a ground track on MSL trends. First, thanks to a rigorous statistical approach, we estimated the error of the MSL bias estimate to link these two missions together, without a calibration phase between Jason-2 and Sentinel-3a. Then, we estimated the impact of this error on the MSL trends versus the period length. Finally, we estimated the impact on MSL trends of the different oceanic variability sampling between the TOPEX historical ground track and the Sentinel-3a. The entire study was conducted both on the global and regional scales.

\section{Estimation of the MSL bias uncertainty}

This part focuses on the estimation of the uncertainty of the relative bias to link MSL time series recorded by two missions. After describing the statistical approach to compute this uncertainty, the results are compared between Jason2/Sentinel-3a and Jason-2/Jason-3.

\subsection{Method to estimate the relative MSL bias uncertainty}

The method to estimate the relative bias between two mean sea level time series is based on the AVISO processing (see www.aviso.altimetry.fr/msl) to link TOPEX-Poseidon (TP) with Jason-1 or Jason-1 with Jason-2. The same method can be applied in the case of Jason-2/Jason-3 to take advantage of the calibration phase between both missions: a common period of nine cycles is selected within the calibration phase, centered on the cycle chosen to switch from Jason-2 data to Jason-3 (usually in the middle of the period). The bias is then computed as the difference of both MSL series averaged over this common period. This method is prevalently used by the MSL groups (AVISO, CU, NOAA, GSFC) with minor differences concerning the choice of the window within the calibration phase (see Masters et al., 2012; Henry et al., 2014).

The calibration phase between Jason- 2 and Jason-3 allows us to make the reasonable assumption that the impact of oceanic variability sampling on each MSL series is negligible because both altimeters measure the same ocean at almost the same time (less than $1 \mathrm{~min}$ ) with respect to the MSL record temporal resolution (about 10 days). It allows maximizing the correlation between Jason-2 and Jason-3 sea surface height (SSH) errors. Indeed, SSH errors are spacetime dependent. Therefore, because Jason-2 and Jason-3 are, during the calibration phase, on the same ground track and spaced less than $1 \mathrm{~min}$ apart, there is a significant correlation between their measurement errors and also between their correction errors (propagation, electromagnetic, geophysical, atmospheric) at the temporal scale of a cycle. Hence, the correlation between Jason-2 and Jason-3 global MSL time series over their calibration phase is strong. In the cases of TP/Jason-1 and Jason-1/Jason-2 calibration phases, we performed an analysis of the MSL time series, showing their correlation was close to 0.8 after removing annual and semiannual signals.

In this study, the main objective is to estimate the relative bias uncertainty it induces. A reliable statistical method has been developed based on simulated Jason-2 and Jason3 MSL data over the calibration phase. The Mercator-Ocean Global Oceanic Reanalysis (GLORYS2v1) has been used. It provides model-based weekly $1 / 4^{\circ}$ grids of sea level anomaly (SLA) over the altimetry era. Jason-2 and Jason-3 simulated MSL data have been generated by interpolating GLORYS SLA bi-linearly (in time and space) on Jason-2 and Jason-3 ground tracks, which - in this paper - are considered identical during the calibration phase. Then, a Jason-2 and a Jason-3 global MSL time series have been computed (based on AVISO MSL method, see www.aviso.altimetry.fr/msl). GLORYS-based simulated MSL data contain however fewer high-frequency signals than real altimetric MSL. In order to 
compute a rigorous estimation of the relative bias uncertainty between Jason-2 and Jason-3, a realistic high-frequency correlated random noise has been designed and added to Jason2 and Jason-3 simulated MSL series. The choice to add this noise directly on the MSL series and not along-track allows us to greatly simplify its design and is sufficient for an accurate relative bias estimation. This noise has two purposes: (i) it reproduces partly the high-frequency signal missing in the original GLORYS-based simulated MSL data, partly the SSH errors, and (ii) it is designed to ensure a high and realistic correlation (close to 0.8) between Jason-2 and Jason-3, simulating their calibration phase. This noise was designed based on the analysis of real altimetric MSL records. Firstly, the variances of Jason-1, Jason-2 and Envisat "real" GMSL time series were estimated. Secondly, a spectral analysis of Jason-1, Jason-2 and Envisat GMSL time series was performed. The noise was then tuned so that the variance and correlation period of the simulated GMSL time series are consistent with these analysis (see Table 1). It is however worth noting that its characteristics strongly impact the results and should be designed carefully. The correlated noise does also not take into account possible drift or offsets (e.g., in the radiometer measurements) that would increase the uncertainty. This choice was made because even though these events are likely to happen, assuming them would be pessimistic.

An estimate of the relative bias is computed from the simulated MSL series over nine cycles. The great interest of this method is to be able to repeat a significant number of times the simulation by changing the random noise (but keeping the high correlation) and the nine cycles of interest within the common period each time (to sample multiple long-period oceanic phenomena, e.g., El Niño). A thousand estimates of the relative bias between Jason-2 and Jason-3 are thus collected. This high number of samples is necessary to reduce the statistical error and provide a significant estimate of the bias uncertainty. After testing the normal distribution of the collection, the uncertainty of the relative bias is computed with 1.96 times the standard deviation, i.e., at $95 \%$ confidence level.

The case of Jason-2 and Sentinel-3a is different because of the absence of calibration phase. However the method to estimate the sea-level bias between both missions is similar to the Jason-2/Jason-3 method with the exception that the common period is not inside a calibration phase. Two uncertainty components are induced by the fact that the ground tracks are different. First, the impact of oceanic variability may no longer be neglected because the two satellites do not observe it with the same space-time sampling. Second, the correlation between SSH errors is reduced because they are space-time dependent. For these two reasons, the correlation between Jason-2 and Sentinel-3a MSL series is significantly smaller than between Jason-2 and Jason-3 ones during their calibration phase. For instance, we performed an analysis of Jason-1 and Envisat "real" GMSL time series, showing their correlation was close to 0.4 after removing annual and semiannual signals.

The method to estimate the relative bias uncertainty also relies on GLORYS-based simulated Jason-2 and Sentinel-3a MSL time series as described previously between Jason-2 and Jason-3, but it is adapted to this configuration without calibration phase, where the impacts of the two uncertainty components - oceanic variability and SSH error decorrelation - are estimated separately. Estimating the impact of oceanic variability comes down to quantify the difference between Jason-2 and Sentinel-3a sea level measures if they were both free from SSH errors. For this, their respective simulated MSL series are compared without addition of highfrequency correlated noises simulating SSH errors: the set of relative bias estimations is only computed by changing the common period. Since Jason-2 and Sentinel-3a have different repetitivity cycles (about 10 days for Jason-2 and 27 days for Sentinel-3a), a 10-day subcycle is used for Sentinel3a GMSL series. The relative bias is again estimated over nine cycles. In the case of the second component, the impact of SSH error decorrelation on the relative bias, the ground tracks need to be identical, but the correlation between the series should be kept low. In other words, two MSL series on the same ground tracks with decorrelated errors are necessary. Therefore the method used for Jason-2/Jason-3 is applied with a correlation between the series monitored around 0.4. The relative bias uncertainties due to each component - oceanic variability or SSH error decorrelation - are then deduced from the standard deviations of their corresponding sets. Note that the total relative bias uncertainty due to the absence of calibration phase is the root sum of squares of its two components.

These methods are designed to estimate the accuracy of the relative bias for Jason-2/Jason-3 or Jason-2/Sentinel-3a for the global MSL time series. The study of regional MSL at the climate scale, however, requires specific regional biases between "reference" missions (Ablain, 2013). Thus, we adapted the methods for the global scale to refine the analyses at the regional scale with a focus on North Atlantic Basin. This region is dominated by mesoscale variability and is thus a challenging choice because it will increase both components of the relative bias uncertainty. The intention here was to estimate an "upper bound" of the uncertainty, but results could be more mitigated in other basins (e.g., East Pacific). However, because of this variability, the correlated noise used for this specific region is also more difficult to design (see Table 1). It should therefore be noted that the results may be slightly degraded by comparison to the global analysis.

\subsection{Results at the global scale}

In this section, the results at the global scale on the estimation of the relative bias accuracy between Jason-2/Jason-3 
Table 1. Characteristics of correlated noise added to simulated mean sea level series at the global scale and in North Atlantic Basin.

\begin{tabular}{lll}
\hline Geographical area & \multicolumn{2}{c}{ Correlated noise characteristics } \\
\cline { 2 - 3 } & $\begin{array}{l}\text { Period of correlation } \\
\text { (days) }\end{array}$ & $\begin{array}{l}\text { Standard deviation } \\
(\mathrm{mm})\end{array}$ \\
\hline Global & 30 & 1.3 \\
North Atlantic Basin & 30 & 5.9 \\
\hline
\end{tabular}

and Jason-2/Sentinel-3a are described; they are synthesized in Table 2.

In the Jason-2/Jason-3 case, simulated data for both satellites provide an uncertainty estimated to $0.9 \mathrm{~mm}$ at $95 \%$ confidence level. This figure may be compared with real measurements during the calibration phase between Jason-1 and Jason-2. As a reminder, the calibration phase between Jason1/Jason-2 lasted 21 cycles from July 2008 to December 2009. By iteratively shifting a nine-cycle window, several (about 10) different estimations of their relative bias were computed as in Ablain et al. (2009). This approach is not statistically significant as the number of samples is very low and samples are correlated. However, this method roughly estimates the Jason-1/Jason-2 relative bias uncertainty to $0.7 \mathrm{~mm}$ with a $95 \%$ confidence, which is in good agreement with the $0.9 \mathrm{~mm}$ estimation obtained with our more accurate method.

Moving on the Jason-2/Sentinel-3a configuration, the relative bias uncertainty is estimated to $2.53 \mathrm{~mm}$ at $95 \%$ confidence level, combining the impact of oceanic variability sampling between the two missions $(0.4 \mathrm{~mm}, 3 \%$ of the global uncertainty) and the SSH error decorrelation $(2.5 \mathrm{~mm}, 97 \%$ of the global uncertainty). This result can also be compared with real data coming from the relative bias from Jason-1 and Envisat, corresponding to a similar configuration with two different ground tracks and without calibration phase between both missions. Since the common era of both satellites is longer than in the Jason-1/Jason-2 case, a significant number of independent relative bias estimates $(\sim 100)$ were computed, providing a more accurate estimation of the uncertainty. Based on Jason-1 and Envisat real global MSL series only, the relative bias uncertainty is estimated to $2.9 \mathrm{~mm}$ at $95 \%$ confidence level, which validates the accuracy of our method based on simulated data.

These results show that linking Sentinel-3a instead of Jason-3 to the "reference" global MSL series raises the uncertainty on the relative bias from 0.9 to $2.5 \mathrm{~mm}$ at $95 \%$ confidence level. This increase is almost completely explained by the fact that, in the absence of calibration phase, the SSH errors of the prior and successor missions are decorrelated. It highlights the importance of calibration phases in the linking of two altimetric missions, allowing a significant reduction of relative bias uncertainties. The impact of this uncertainty level on the MSL estimation is described further in the Sect. 3.

\subsection{Results at the regional scale}

After describing results at the global scale, similar analyses have been performed at the regional scale. Hereafter, results are focused on the North Atlantic Basin, estimating the relative bias accuracy for Jason-2/Jason-3 or Jason-2/Sentinel$3 a$. Table 3 synthesizes the results. Applying exactly the same method but with data selected only in the North Atlantic Basin, the Jason-2/Jason-3 relative bias uncertainty is estimated to $5.2 \mathrm{~mm}$ at $95 \%$ confidence level. Results show that, even with a calibration phase, the uncertainty on the Jason2/Jason-3 relative bias is high in the North Atlantic Basin. Indeed, this basin has high mesoscale variability (Le Traon et al., 1990); therefore, the MSL contains stronger highfrequency signals than at the global scale. This directly impacts the uncertainty on the relative bias, even if the correlation between Jason- 2 and Jason-3 is strong over the calibration phase. As at the global scale, this figure is compared with the estimation of the Jason-1/Jason-2 relative bias uncertainty from real measurements. A lower level of uncertainty close to $2.2 \mathrm{~mm}$ at $95 \%$ confidence level is obtained in this case. However, as explained in the previous section, the Jason-1/Jason-2 bias uncertainty with real measurements is underestimated because the calibration phase is too short, and therefore not statistically significant, particularly with the high variance of the signal in this basin.

The Jason-2/Sentinel-3a relative bias uncertainty in North Atlantic Basin is estimated to $11.3 \mathrm{~mm}$ at $95 \%$ confidence level; $2 \%$ is due to the impact of oceanic variability $(1.7 \mathrm{~mm})$, and the remaining $98 \%$ is attributed to sea surface height error decorrelation $(11.2 \mathrm{~mm})$. Results suggest a high uncertainty in this basin which is partly explained by the high oceanic variability as well as by the low correlation between Jason-2 and Sentinel-3a series in the absence of calibration phase. The contribution of each component of the uncertainty is fully consistent with global results. As in the previous section, the uncertainty on the relative bias for Jason-1/Envisat with real measurements has been estimated in the North Atlantic Basin for comparison. With this alternative method, we obtained a $9.1 \mathrm{~mm}$ uncertainty at $95 \%$ confidence level, which is in good agreement with our result. The small difference may however be explained by the fact that Envisat and Sentinel-3a ground tracks - i.e., their space-time sampling of oceanic variability - are also different.

\section{Impact of MSL bias uncertainties on MSL trends}

After estimating the MSL bias uncertainties for Jason2/Jason-3 and Jason-2/Sentinel-3a, the impact on MSL evolution is thoroughly analyzed in this section.

\subsection{Method}

The MSL relative bias uncertainty between two missions has been expressed as an instantaneous error in the MSL time se- 
Table 2. Jason-2/Jason-3 and Jason-2/Sentinel-3a relative bias uncertainties on the global mean sea level.

\begin{tabular}{llll}
\hline Case (global) & \multicolumn{3}{c}{ Relative bias uncertainty (mm) } \\
\cline { 2 - 4 } & $\begin{array}{l}\text { Impact of oceanic variability } \\
\text { sampling only }\end{array}$ & $\begin{array}{l}\text { Impact of SSH errors } \\
\text { decorrelation only }\end{array}$ & $\begin{array}{l}\text { Total } \\
\text { uncertainty }\end{array}$ \\
\hline Jason-2/Jason-3 & 0 & 0.9 & 0.9 \\
Jason-2/Sentinel-3a & 0.4 & 2.5 & 2.53 \\
\hline
\end{tabular}

Table 3. Jason-2/Jason-3 and Jason-2/Sentinel-3a relative bias uncertainties on the North Atlantic mean sea level.

\begin{tabular}{llll}
\hline Case (North Atlantic) & \multicolumn{3}{c}{ Relative bias uncertainty (mm) } \\
\cline { 2 - 4 } & $\begin{array}{l}\text { Impact of oceanic variability } \\
\text { sampling only }\end{array}$ & $\begin{array}{l}\text { Impact of SSH errors } \\
\text { decorrelation only }\end{array}$ & $\begin{array}{l}\text { Total } \\
\text { uncertainty }\end{array}$ \\
\hline Jason-2/Jason-3 & 0 & 5.2 & 5.2 \\
Jason-2/Sentinel-3a & 1.7 & 11.2 & 11.3 \\
\hline
\end{tabular}

ries. For climate studies, it is important to take it into account in MSL trend uncertainties (Ablain et al., 2009).

The method developed in this study is based on the ordinary least squares method in the case of a linear regression to Heaviside functions. The Heaviside function models the uncertainty due to a mission change: 0 before the change and the amplitude of the bias uncertainty afterwards. The trend uncertainty is then given as a function of time, highly dependent on the duration of the time series before the mission change $\left(t_{\mathrm{c}}\right)$, and the amplitude of the instantaneous uncertainty; see Eq. (1) and demonstration in the Supplement, where $t$ is time (zero at the start of the series), $b_{\mathrm{u}}$ the bias uncertainty, $t_{\mathrm{c}}$ the time of mission change, and $p$ the sampling period (9.91 days for Jason and 27 days for Sentinel-3a, therefore in our case $p \ll t_{\mathrm{c}}$ ). The trend uncertainty has a null value before $t_{\mathrm{c}}$, then increases rapidly to reach a maximum at time $1.5 \times t_{\mathrm{c}}$ (assuming $p \ll t_{\mathrm{c}}$ ) and finally decreases slowly and converges towards an asymptote at 0 . In other words, when a new mission is added to the "reference" MSL series, the relative bias uncertainty raises the trend uncertainty at first but is then compensated for by the addition of new data.

Trend uncertainty $(t)=$

$\frac{6 \cdot b_{\mathrm{u}} \cdot t_{C}\left(t-t_{\mathrm{c}}\right)}{t\left(t^{2}-p^{2}\right)}$ for $t \geq t_{\mathrm{c}}, 0$ for $t<t_{\mathrm{c}}$

Equation (1) applies to the case of only one mission change. However, in the "reference" MSL series, there are several mission changes: TOPEX-A/TOPEX-B, TOPEXB/Jason- 1 and Jason-1/Jason-2. In the present study, another mission change has been added: Jason-2/Jason-3 or Jason2/Sentinel-3a. With several mission changes, Eq. (1) becomes more complex and the analytic formula has not been derived. The general behavior of the trend uncertainty remains similar but strongly depends on the length of the analysis period.
Sea level climate change initiative requirements on MSL trend are based on a 10-year period (GCOS 2011). We thus based our analysis on a 10-year period, 2011-2020, centered on the mission change from Jason-2 to Jason-3 or from Jason-2 to Sentinel-3a. Since the comparison requires a common date, we assumed the mission change occurs in January 2016 for both missions. Changing the date would change the figures but not the conclusions of the study, as Eq. (1) is directly proportional to the bias uncertainty and all other parameters are taken equal. However, the derivative of Eq. (1) with respect to $t_{\mathrm{c}}$ shows that the impact of the bias uncertainty on the 10-year linear regression is maximized when the mission change happens in the middle of the period, after 5 years.

The analysis has also been extended to a 15 -year period to include the impact of Jason-1/Jason-2 mission change, and to a 25-year period to include TOPEX-A/TOPEX-B and TOPEX-B/Jason-1 mission changes. These last results are illustrative in order to well understand the dependence between the period length and the trend uncertainty. Relative bias uncertainties between Jason-1/Jason-2 and TOPEXB/Jason- 1 are considered equal to the global and regional estimates of the Jason-2/Jason-3 uncertainty estimated in this study as the cases are theoretically equivalent. For TOPEXA/TOPEX-B, Ablain et al. (2009) estimate the uncertainty is twice the TOPEX-B/Jason-1 uncertainty. Thus, we used $2 \mathrm{~mm}$ in the global case and $1 \mathrm{~cm}$ in the North Atlantic case. This approximation may potentially reduce the accuracy of the diagnosis on the 25-year period in this basin.

\subsection{Impact at the global scale}

Figure 1 shows the impact of inter-mission relative bias uncertainty on the estimation of the global MSL trend in time. The analysis is focused on a 10-year period (upper panel). Results show the trend uncertainty is raised by $0.14 \mathrm{~mm} \mathrm{yr}^{-1}$ 


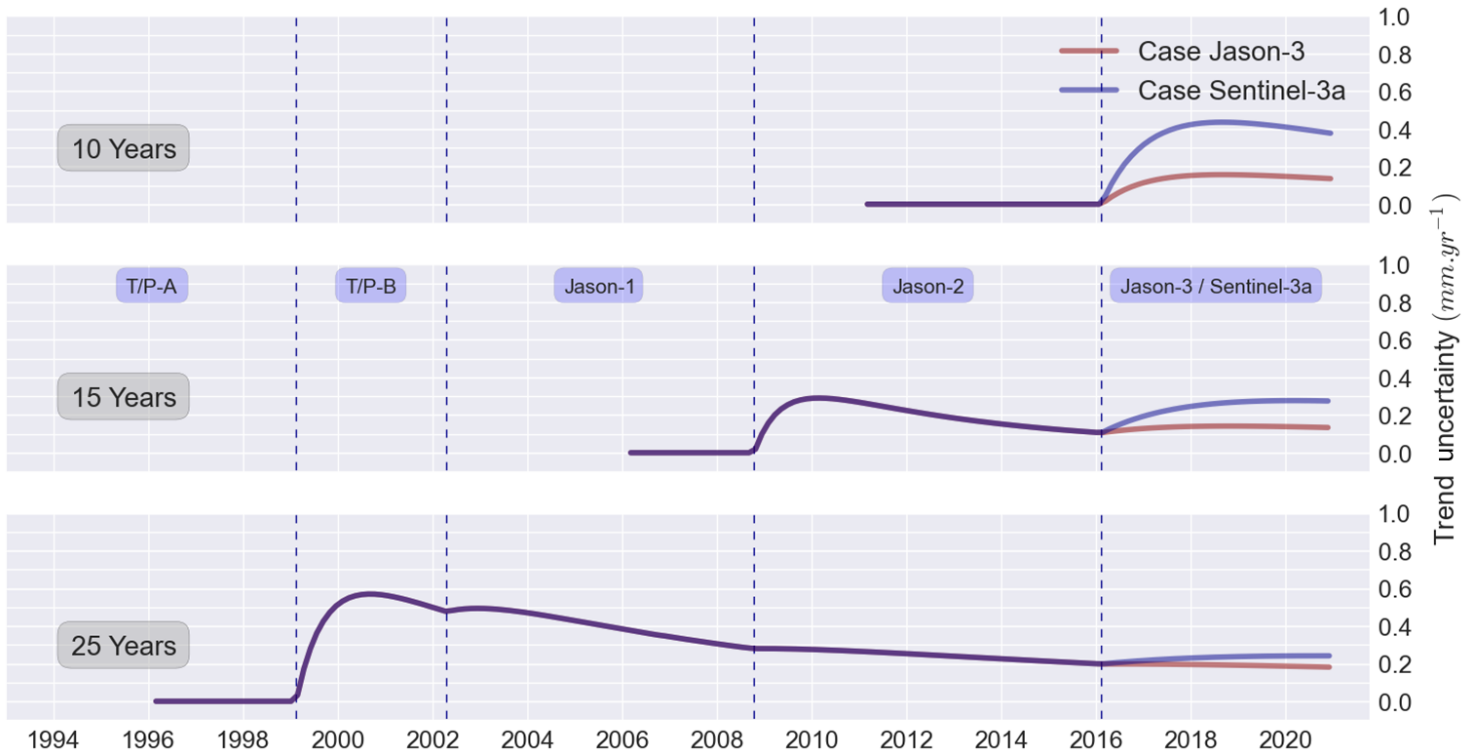

Figure 1. Impact of global mean sea level inter-mission linking bias uncertainties on the estimation of the MSL trend over 10 years (upper panel), 15 years (middle panel), and 25 years (lower panel), in the cases of Jason-3 and Sentinel-3a.

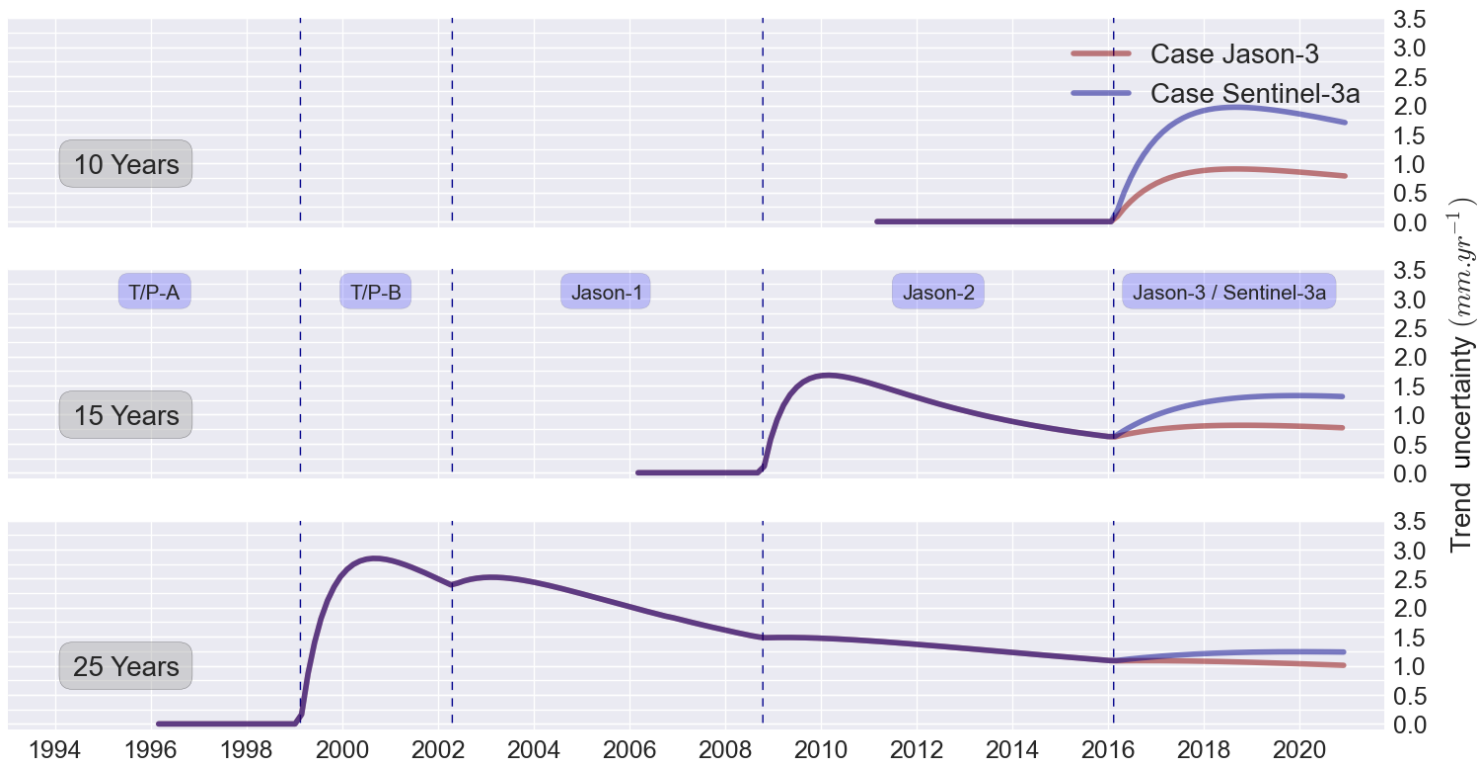

Figure 2. Impact of North Atlantic mean sea level inter-mission linking bias uncertainties on the estimation of the MSL trend over 10 years (upper panel), 15 years (middle panel), and 25 years (lower panel), in the cases of Jason-3 and Sentinel-3a.

- when Jason-3 is linked to Jason-2 - and $0.38 \mathrm{~mm} \mathrm{yr}^{-1}$ when Sentinel-3a is linked to Jason-2. Climate users require an uncertainty less than $0.3 \mathrm{~mm} \mathrm{yr}^{-1}$ over 10 years (GCOS, 2011) on the global MSL trend. Using Sentinel-3a instead of Jason-3 would therefore already exceed user requirements, even though relative bias uncertainty is only one component of the global MSL error budget (Ablain et al., 2009).

With longer time series including other mission changes (see Fig. 1, middle and lower panels), the large number of samples brings stability to the trend estimation at the end of the period. Even though the Jason-2/Sentinel-3a relative bias uncertainty is larger than for Jason-2/Jason-3, differences are reduced. However, Sentinel-3a increases the trend uncertainty significantly, by $0.15 \mathrm{~mm} \mathrm{yr}^{-1}$ over 15 years and almost $0.1 \mathrm{~mm} \mathrm{yr}^{-1}$ over 25 years, by comparison to Jason3. These differences are significant considering global MSL error budget methods usually estimate the trend uncertainty 


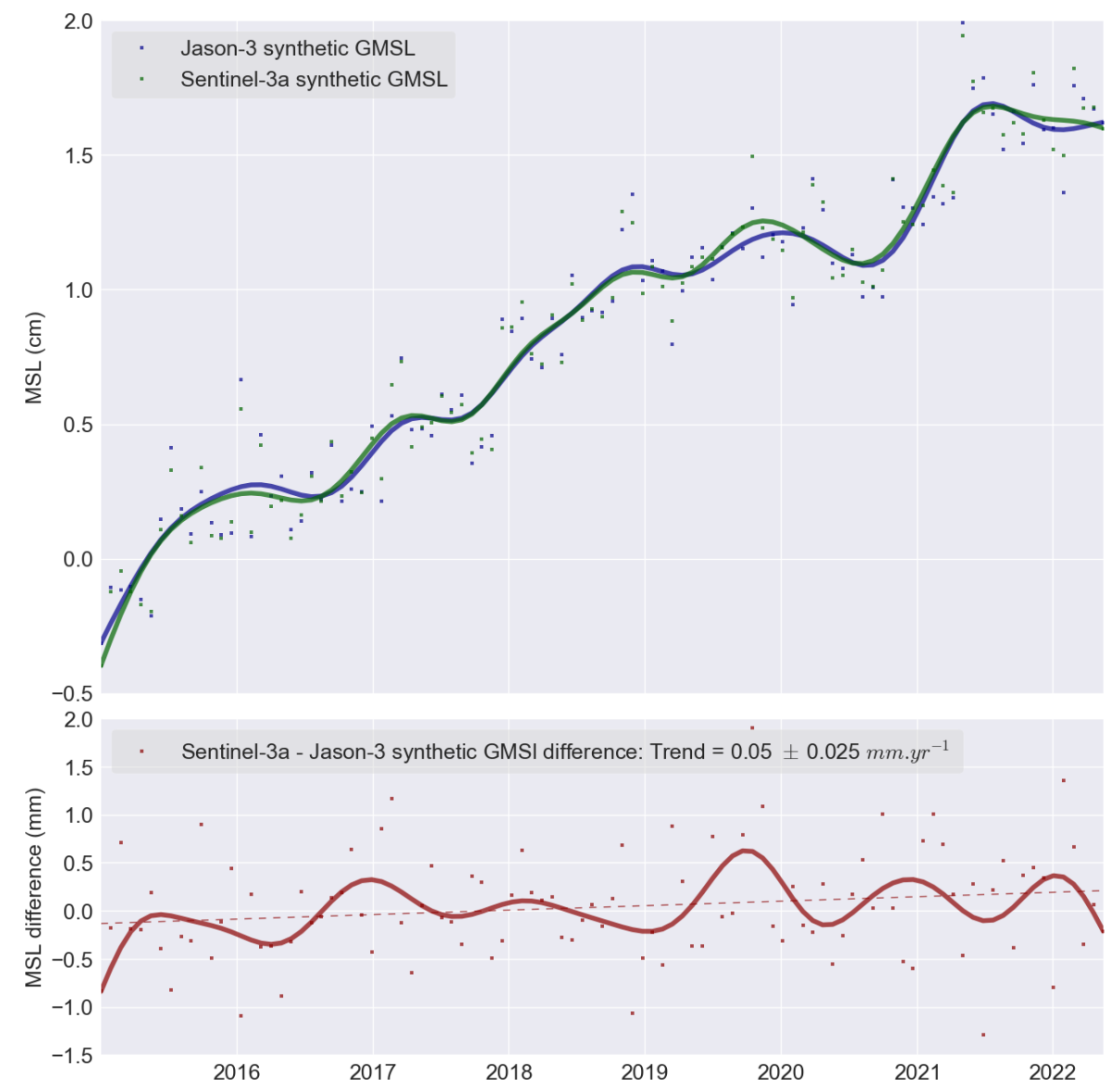

Figure 3. Long-term impact of changing from TOPEX "historical" to Sentinel-3a ground tracks on the global mean sea level evolutions.

close to $0.5 \mathrm{~mm} \mathrm{yr}^{-1}$ (Ablain et al., 2015; Nerem et al., 2010) over a period longer than 10 years.

\subsection{Impact at the regional scale}

Figure 2 shows the impact of inter-mission relative bias uncertainty on the estimation of the North Atlantic Basin MSL trend in time. Over a 10-year period (upper panel), results show the trend uncertainty reaches $0.78 \mathrm{~mm} \mathrm{yr}^{-1}$ in 2020 when Jason-3 is linked to Jason-2 and $1.70 \mathrm{~mm} \mathrm{yr}^{-1}$ in 2020 when Sentinel-3a is linked to Jason-2. The differences between both cases are reduced to $0.60 \mathrm{~mm} \mathrm{yr}^{-1}$ over 15 years (middle panel) and $0.25 \mathrm{~mm}$ over 25 years (lower panel). Climate users require an uncertainty less than $1 \mathrm{~mm} \mathrm{yr}^{-1}$ on the MSL trend over 10 years in a $2^{\circ} \times 2^{\circ}$ box (GCOS, 2011). The difference of areas between a $2^{\circ} \times 2^{\circ}$ box and the North Atlantic Basin does not allow a direct comparison between results and user requirements. Nevertheless, it gives an upper bound of the order of magnitude that may be required. Linking Sentinel-3a instead of Jason-3 to Jason-2 induces an increase of the trend uncertainty over 10 years by almost $1 \mathrm{~mm} \mathrm{yr}^{-1}$ and is therefore very significant.

\section{Impact of oceanic variability sampling on MSL trends}

Linking the Sentinel-3a MSL series to the "reference" MSL induces not only an uncertainty on the relative bias but also a long-term error due to the fact that the space samplings are not consistent between the Sentinel-3a orbit and the "historical" TOPEX one. However, Jason-3 mission is on the "historical" orbit. Hence linking Jason-3 to Jason-2 does not induce a long-term error. In this part, this long-term error, which also impacts the trend uncertainty, is estimated and described at the global and the regional scale.

\subsection{Method}

Basically, solving this issue is the same as estimating the trend difference between Sentinel-3a and Jason-3 over the same period. For this, we used synthetic GLORYS-based sea level anomaly on Sentinel-3a and Jason-3 ground tracks. After computing the corresponding global MSL time series (AVISO method, see AVISO website), the global long-term error is estimated as the trend of the difference between the two series. The regional long-term error is also estimated 


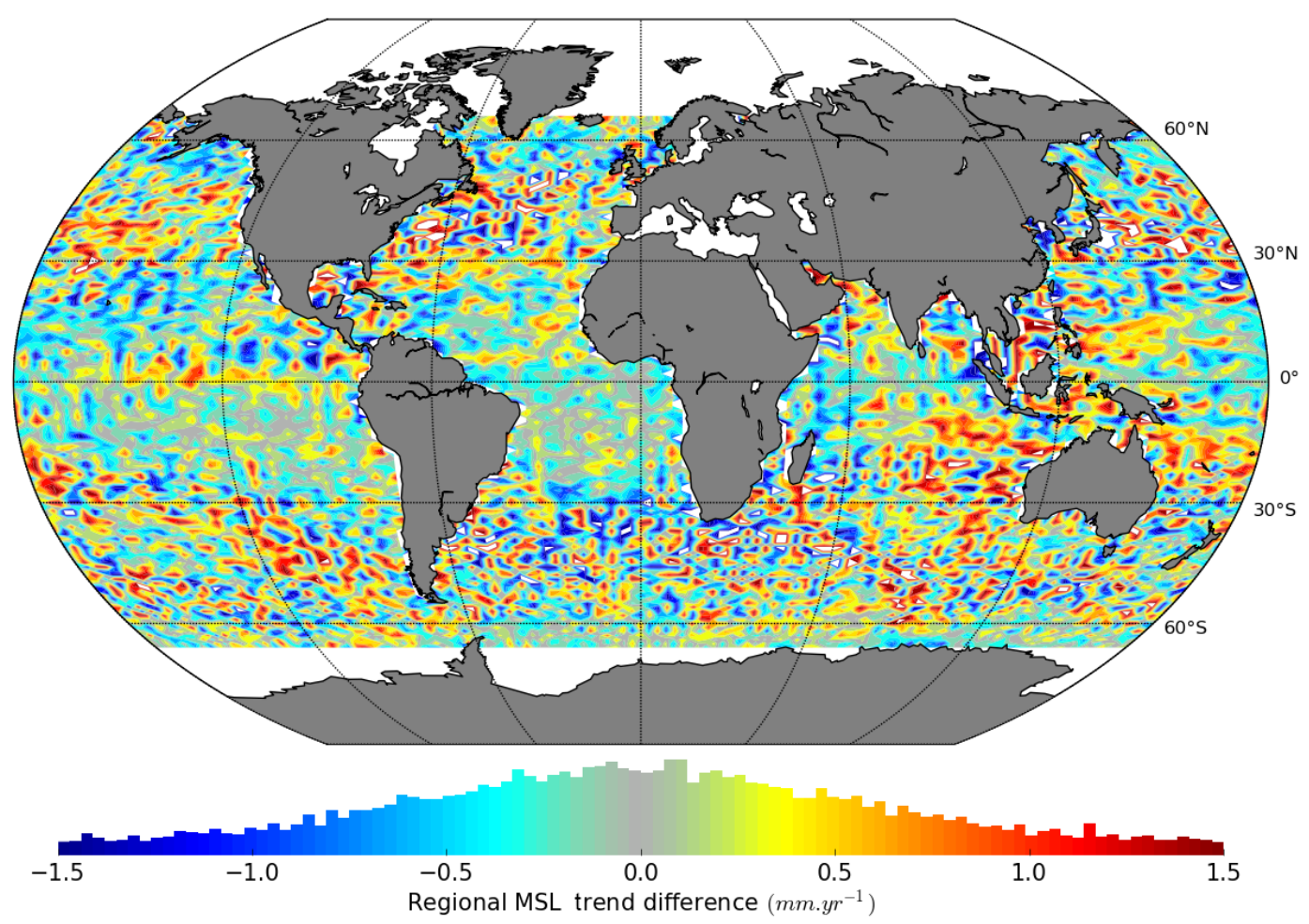

Figure 4. Long-term impact of changing from TOPEX "historical" to Sentinel-3a ground tracks on the local mean sea level trend.

by computing the map of the trend of both Sentinel-3a and Jason-3 MSL differences in $3^{\circ} \times 1^{\circ}$ boxes.

\subsection{Impact at the global scale}

Figure 3 represents Jason-3 and Sentinel-3a simulated global MSL series without annual and semi-annual signals (upper panel) and their difference (lower panel). Results show the mid-term evolution differences reach 1 to $1.5 \mathrm{~mm}$ locally with a $0.28 \mathrm{~mm}$ standard deviation. The impact on midterm evolutions is therefore small but significant. Similarly, a $0.05 \pm 0.025 \mathrm{~mm} \mathrm{yr}^{-1}$ trend is estimated on the difference between Sentinel-3a and Jason-3 over the 7 years. Thus, the impact of ground tracks on GMSL long-term evolutions is not negligible but small compared to the impact of linking uncertainty.

\subsection{Impact at the regional scale}

The differences between Sentinel-3a and Jason-3 regional simulated MSL trends over a 7-year period are represented in Fig. 4. Differences exceed $1.5 \mathrm{~mm} \mathrm{yr}^{-1}$ in regions of high oceanic variability (Kuroshio, Agulhas, ACC, Falklands, etc.). However, the associated regression errors are particularly large (up to $3 \mathrm{~mm} \mathrm{yr}^{-1}$, not shown) in these regions due to eddies and high-frequency signals. The histogram below the map shows only $70 \%$ of MSL trend differences are less than $1 \mathrm{~mm} \mathrm{yr}^{-1}$, which corresponds to the cli- mate user requirements for regional MSL trend uncertainty (GCOS, 2011). Though results must be balanced by the corresponding regression errors - about $0.5 \mathrm{~mm} \mathrm{yr}^{-1}$ - they suggest the regional impact of linking Jason-2/Sentinel-3a instead of Jason-2/Jason-3 prevents meeting user requirements on regional MSL trend uncertainties.

\section{Conclusions}

This paper aims at considering the possibility that the "reference" MSL series - based on TOPEX/Poseidon, Jason-1, and Jason-2 missions - could be extended with another mission on a different orbit and with a similar error budget: Sentinel3a instead of Jason-3. Two potential consequences have been identified and investigated by comparing them with the situation in which Jason-3 succeeded Jason-2 in the reference MSL time series. The impact on the MSL time series has been quantified by separating the instantaneous uncertainty due to the absence of calibration phase, which impacts the trend uncertainty, from the long-term uncertainty due to the change of ground track.

Results show calibration phases play a crucial role in the accuracy of the linking between the reference and Jason-3 MSL, both at the global and regional scales. With a calibration phase, the uncertainty on the relative bias is $0.9 \mathrm{~mm}$, at the $95 \%$ confidence level, on the global MSL (GMSL). The corresponding impact on the trend is small though sig- 
nificant: $0.1 \mathrm{~mm} \mathrm{yr}^{-1}$ over 10 years. Without a calibration phase, however, the uncertainty on the relative bias between the reference GMSL and Sentinel-3a GMSL is increased to $2.5 \mathrm{~mm}$ at the $95 \%$ confidence level. This uncertainty induces a global MSL trend uncertainty of $0.4 \mathrm{~mm} \mathrm{yr}^{-1}$ over 10 years. At a regional scale, in North Atlantic Basin, Jason2/Jason-3 and Jason-2/Sentinel-3a relative bias uncertainties induce respectively 0.78 and $1.70 \mathrm{~mm} \mathrm{yr}^{-1}$ trend uncertainties over 10 years at $95 \%$ confidence level.

The difference between the Jason-2/Jason-3 and Jason2/Sentinel-3a uncertainties is mainly explained by the decorrelation of SSH errors in the absence of calibration phase for Sentinel-3a. This stresses the importance of calibration phases for an accurate computation of the MSL trend.

Moreover, the differences between the TOPEX/Jason historical ground track and the Sentinel-3a ground track induce another $0.05 \mathrm{~mm} \mathrm{yr}^{-1}$ uncertainty on the Jason-2/Sentinel-3a MSL long-term evolution over 7 years.

The main conclusion is that linking the Sentinel-3 MSL time series to Jason-2 has a strong impact on the global and regional MSL uncertainty. The climate user requirements (GCOS, 2011) require an uncertainty in the MSL trend of less than $0.3 \mathrm{~mm} \mathrm{yr}^{-1}$ at the global scale and $1 \mathrm{~mm} \mathrm{yr}^{-1}$ in $2^{\circ} \times 2^{\circ}$ boxes over 10 years.

As far as possible, the altimetric missions linked to the reference MSL record should be on the historical TOPEX/Jason orbit to minimize the error on the MSL trend. Otherwise, a significant uncertainty will be associated with the intermission bias, resulting in an uncertainty on the trend that will exceed user requirements. This conclusion is however directly related to the method used to link the missions. In this paper, it is assumed that Sentinel-3a would be linked to Jason-2 (or Jason-3) with the same methodology that is currently the most accurate and is widely used by the prominent MSL computation groups (see Masters et al., 2012; Henry et al., 2014). New methods could potentially be designed to reduce this uncertainty, but the challenge is to find a method that uses a very limited period to avoid possible drifting issues (e.g., of the radiometer) between the consecutive missions. Using external MSL series (e.g., tidal gauges or a third mission) as a reference is also challenging because it introduces new sources of errors and uncertainties.

The inter-mission relative bias uncertainties estimated in this paper also contribute to better modeling error-covariance matrices of the continuous MSL record. With an accurate model of the MSL error budget, it is possible to access a very accurate estimate of the trend and most importantly its uncertainty, using a generalized least squares approach (see Ablain et al., 2009).

The Supplement related to this article is available online at doi:10.5194/os-12-9-2016-supplement.
Acknowledgements. This work was performed in the framework of the ESA CCI program supported by ESA. This work was also made possible thanks to the support of CNES for several years in altimetry data processing, in particular in the framework of the SALP project (Service d'Altimétrie et de Localisation Précise). We would also like to thank J. Benveniste and N. Picot for their diligent review of this study.

GLORYS reanalysis received support from INSU-CNRS, Mercator Océan, Groupe Mission Mercator Coriolis and the European Community's Seventh Framework Programme FP7/2007-2013 under grant agreement no. 218812 (MyOcean)

Edited by: J. M. Huthnance

\section{References}

Ablain, M.: Validation Report:WP2500 Regional SSH Bias Corrections between Altimetry Missions, http://www.esa-sealevel-cci. org/PublicDocuments/SLCCI-ValidationReport_WP2500_

AltimetrySSHBiasBetweenMissions.docx (last access: 4 January 2016), 2013.

Ablain, M., Cazenave, A., Valladeau, G., and Guinehut, S.: A new assessment of the error budget of global mean sea level rate estimated by satellite altimetry over 1993-2008, Ocean Sci., 5, 193201, doi:10.5194/os-5-193-2009, 2009.

Ablain, M., Cazenave, A., Larnicol, G., Balmaseda, M., Cipollini, P., Faugère, Y., Fernandes, M. J., Henry, O., Johannessen, J. A., Knudsen, P., Andersen, O., Legeais, J., Meyssignac, B., Picot, N., Roca, M., Rudenko, S., Scharffenberg, M. G., Stammer, D., Timms, G., and Benveniste, J.: Improved sea level record over the satellite altimetry era (1993-2010) from the Climate Change Initiative project, Ocean Sci., 11, 67-82, doi:10.5194/os-11-672015, 2015.

Berger, M., Moreno, J., Johannessen, J. A., Levelt, P. F., and Hanssen R. F.: ESA's Sentinel Missions in Support of Earth System Science, Remote Sens. Environ., 120, 84-90, doi:10.1016/j.rse.2011.07.023, 2012.

Cazenave, A.: Present-Day Sea Level Change: Observations and Causes, Rev. Geophys., 42, RG3001, doi:10.1029/2003RG000139, 2004.

Dibarboure, G., Pujol, M.-I., Briol, F., Le Traon, P. Y., Larnicol, G., Picot, N., Mertz, F., and Ablain, M.: Jason-2 in DUACS: Updated System Description, First Tandem Results and Impact on Processing and Products, Mar. Geod., 34, 214-241, 2011.

Donlon, C., Berruti, B., Buongiorno, A., Ferreira, M.-H., Féménias, P., Frerick, J., Goryl, P. et al.: The Global Monitoring for Environment and Security (GMES) Sentinel-3 Mission, Remote Sens. Environ., 120, 37-57, doi:10.1016/j.rse.2011.07.024, 2012.

Dorandeu, J., Ablain, M., and Le Traon, P.-Y.: Reducing CrossTrack Geoid Gradient Errors around TOPEX/Poseidon and Jason-1 Nominal Tracks: Application to Calculation of Sea Level Anomalies, J. Atmos. Ocean. Tech., 20, 1826-1838, 2003.

Dorandeu, J., Ablain, M., Faugere, Y., Mertz, F., Soussi, B., and Vincent, P.: Jason-1 Global Statistical Evaluation and Performance Assessment: Calibration and Cross-Calibration Results, Mar. Geod., 27, 345-372, doi:10.1080/01490410490889094, 2004. 
GCOS: Systematic Observation Requirements For Satellite-Based Data Products for Climate, https://www.wmo.int/pages/prog/ gcos/Publications/gcos-154.pdf (last access: 4 January 2016). 2011.

Henry, O., Ablain, M., Meyssignac, B., Cazenave, A., Masters, D., Nerem, S., and Garric, G.: Effect of the Processing Methodology on Satellite Altimetry-Based Global Mean Sea Level Rise over the Jason-1 Operating Period, J. Geodesy, 88, 351-361, 2014.

Larnicol, G., Cazenave, A., Faugère, Y., Ablain, M., Johannessen, J., Stammer, D., Timms, G., Knudsen, P., Cipolini, P., Roca, M., Rudenko, S., Fernandes, J., Balmaseda, M., Guinle, T., and Benveniste, J.: Two Decades of Global and Regional Sea Level Observation from the ESA Climate Change Initiative Sea Level Project, OSTST, http://www.aviso.altimetry.fr/fileadmin/documents/OSTST/ 2013/oral/Ablain_Pres_OSTST2013_SLCCI.pdf (last access: 4 January 2016), 2013.

Le Traon, P. Y., Rouquet, M. C., and Boissier, C.: Spatial Scales of Mesoscale Variability in the North Atlantic as Deduced from Geosat Data, J. Geophys. Res., 95, 20267, doi:10.1029/JC095iC11p20267, 1990.

Leuliette, E. W., Steven Nerem, R., and Mitchum, G. T.: Calibration of TOPEX/Poseidon and Jason Altimeter Data to Construct a Continuous Record of Mean Sea Level Change, Mar. Geod., 27, 79-94, doi:10.1080/01490410490465193, 2004.
Masters, D., Nerem, R. S., Choe, C., Leuliette, E., Beckley, B., White, N., and Ablain, M.: Comparison of Global Mean Sea Level Time Series from TOPEX/Poseidon, Jason-1, and Jason-2, Mar. Geod., 35(sup1), 20-41, doi:10.1080/01490419.2012.717862, 2012.

Nerem, R. S., Chambers, D. P., Choe, C., and Mitchum, G. T.: Estimating Mean Sea Level Change from the TOPEX and Jason Altimeter Missions, Mar. Geod., 33, Taylor \& Francis Group, 435-446, doi:10.1080/01490419.2010.491031, 2010.

Ollivier, A., Faugere, Y., Picot, N., Ablain, M., Femenias, P., and Beneveniste, J.: Envisat Ocean Altimeter Becoming Relevant for Mean Sea Level Trend Studies, Mar. Geod., 35(sup1), 118-136, doi:10.1080/01490419.2012.721632, 2012.

Philipps, S., Desai, S., Roinard, H., Ablain, M., and Valladeau, G.: Global Jason-1\&2 Quality Assessment, OSTST, http://www.aviso.altimetry.fr/fileadmin/documents/OSTST/ 2013/oral/Philipps_Calval_Jason.pdf, 2013.

Prandi, P., Ablain, M., Cazenave, A., and Picot, N.: A New Estimation of Mean Sea Level in the Arctic Ocean from Satellite Altimetry, Mar. Geod., 35(sup1), 61-81, doi:10.1080/01490419.2012, 2012. 